\title{
Simplified method to measure glucocorticoid metabolites in faeces of horses
}

\author{
Birgit Flauger ${ }^{1}$, Konstanze Krueger ${ }^{1}$, Hartmut Gerhards², Erich Möstl ${ }^{3}$ \\ corresponding author: Birgit Flauger: info@Birgit-Flauger.de \\ ${ }^{1}$ Biology I, Institute of Zoology, University of Regensburg, 93040 Regensburg, Germany \\ ${ }^{2}$ Equine Clinic, Faculty of Veterinary Medicine, Ludwig-Maximilians-University Munich, Ve- \\ terinärstraße 13, 80539 München, Germany \\ ${ }^{3}$ Institute of Biochemistry, Department of Biomedical Sciences/Biochemistry, University of \\ Veterinary Medicine, Veterinär-Platz 1, 1210 Vienna, Austria
}

'The original publication is available at www.springerlink.com' 2010

Veterinary Research Communications DOI: 10.1007/s11259-010-9344-y

\section{Abstract}

Glucocorticoids or their metabolites can be measured in several body fluids or excreta, including plasma, saliva, urine and faeces. In recent years the measurement of glucocorticoid metabolites (GCMs) in faeces has gained increasing attention, because of its suitability for wild populations. In horses, however, the group-specific enzyme immunoassay described so far has a limited practicability due to its complex extraction procedure. Therefore, we tested the applicability of other enzyme immunoassays for glucocorticoid metabolites. The present study clearly proved that an enzyme immunoassay (EIA) for 11-oxoaetiocholanolone using 11-oxoaetiocholanolone-17-CMO: BSA (3a,11-oxo-A EIA) as antigen showed high amounts of immunoreactive substances. Therefore it was possible to use just a small amount of the supernatant of a methanolic suspension of faeces. The results correlated well with the already described method for measuring GCMs in horse faeces, i.e. analysing the samples with an EIA after a two step clean up procedure of the samples (Merl et al. 2000). In addition, the 3a,11-oxo-A EIA has the advantage of providing a bigger difference between baseline values and peak values after ACTH stimulation. The new assay increased the accuracy of the test, lowered the expenses per sample, and storing samples at room temperature after collection was less critical than with other assays investigated in our study. This is a big advantage both in the field of wildlife management of equids and in the field of equestrian sports and it shows the importance of choosing an assay which is in good accordance with the metabolites excreted in a given species.

\section{Keywords}

ACTH challenge, enzyme immunoassay, stress behaviour, cortisol

\section{Introduction}

In vertebrates, the frontline hormones in stressful situations are glucocorticoids and catecholamines. Their increased secretion enhances adaptive physiological responses (Wingfield and Ramenofsky 1999; Sapolsky et al. 2000). The two main "stress-axes" involved are the autonomic nervous system (Cannon 1935) and the hypothalamo-pituitary-adrenocortical (Selye 1936) axes.

Glucocorticoids, or their metabolites, can be measured in several body fluids or excreta, including plasma, saliva, urine and faeces (e.g. Möstl and Palme 2002). The cortisol in blood can be divided into a free fraction and a fraction bound to corticoid binding globulins (Matteri et al. 2000), free cortisol representing the biologically active form (Moons et al. 2002). Stress reduces the binding capacity of the corticosteroid binding globulin (Alexander and Irvine 1998). Traditionally, plasma has been used, but sample collection is difficult and stressful for the animals, which may confound the results (Hopster et al. 1999).

Therefore, in recent years the measurement of glucocorticoid metabolites (GCMs) in faeces has gained increasing attention for wild populations (Heistermann et al. 2006), wildlife management, and conservation as well as behavioural biology (Möstl and Palme 2002; Touma and Palme 2005), largely because it uses a non-invasive and feedback-free sampling method. 
Even though horses excrete $41 \%$ of radioactive cortisol via faeces (Palme et al. 1996) which should therefore be well suited for detecting GCMs in faeces, the extraction procedure for horse faeces described so far (Merl et al. 2000; Gorgasser et al. 2007) is more complicated than in other species. This is due to the fact that the assay used picks up only metabolites which are present in minor amounts, but not the dominant ones.

Within the framework of a large project to physiologically validate cortisol and GCM secretion in horses, we tried to find an assay which cross reacts in a higher extent with the GCMs in horse faeces.

\section{Material and Methods}

\section{Animals}

A total of ten horses (five mares, five stallions) were used for the experiment, eight warmblood horses, one haflinger and one pony, all aged between three and 14 years. The experiment was conducted at the veterinarian department of the Ludwig-MaximiliansUniversity in Munich, where the horses were stabled at least three days prior to the testing. The horses were kept in individual boxes with a bedding of straw and were turned out on paddocks during the day. The daily feed of the horses was composed of hay ad libitum and a compound feed twice a day. The animal experiment was permitted by the Bavarian Government (reference number 55.2-1-54-2531-121-07).

\section{$\underline{\text { ACTH Challenge Test }}$}

The horses were tested in three batches consisting of three, three and four horses, respectively. The sampling procedure differed slightly between these experiments because of adjustments to the horses turn-out times.

The stimulation test was conducted over a period of six days. On day 1 and 2, blood and faecal samples were taken in the morning. On day 3 only faecal samples were taken in the morning, and blood samples soon after inserting a permanent catheter into the vena jugularis. Two hours after the catheterization $0.05 \mathrm{mg} / 100 \mathrm{kgBM}$ ACTH were injected i.m. (preparation: Synacthen Injektionslösung ${ }^{\circledR}$, Novartis Pharma $\mathrm{GmbH}$, Nuremberg). Blood samples were taken 30, 60, 90, 120 and 240 min after ACTH application. In the evening of the same day blood and faecal samples were collected again. Thereafter the permanent catheter was removed. On day 4, 5 and 6 blood and faecal samples were taken in the morning and in the evening.
On day 7 , after faecal sample collection in the morning, $16 \mathrm{mg} / 100 \mathrm{kgBM}$ dexamethasone (preparation: Dexamethason-Injektionslösung ${ }^{\circledR}$, CP-Pharma Handelsges. mbH, Burgdorf) were injected i.m. After 90 min blood samples were taken. On the same day blood and faeces were collected again in the evening. On day 8,9 and 10 blood and faecal samples were taken in the morning.

\section{Sample Processing}

Blood samples were taken with a heparin Monovette ${ }^{8} 2 \mathrm{ml} \mathrm{LH}$, Sarstedt, and immediately centrifuged for $8 \mathrm{~min}$ at $1000 \mathrm{~g}$. The plasma was stored at $-20^{\circ} \mathrm{C}$. During the 30 min intervals of the ACTH stimulation the blood samples were kept on ice and centrifuged within 2 hours. Plasma cortisol levels were analysed using an EIA as described by Palme and Möstl (1997).

Faecal samples were collected with one-way gloves, stored in glasses and immediately frozen at $-20^{\circ} \mathrm{C}$, or kept on ice until freezing. For processing faecal samples two different protocols were used and afterwards the samples were analysed by different groupspecific EIAs. The first protocol (extraction method described by Merl et al. 2000) is a two step extraction including addition of water/methanol. After centrifugation, the supernatant is diluted with $\mathrm{NaHCO} 3$ and reextracted with diethyl ether. The organic solvent has to be evaporated and the residue redissolved in assay buffer before performing the EIA. Using assays which cross reacted with the faecal GCMs of horses in a higher amount than the assay already described; we extracted horse faeces as described for faecal GCM extraction in ruminants (Palme and Möstl 1997). In brief, $0.5 \mathrm{~g}$ faeces plus $1 \mathrm{ml}$ water and $4 \mathrm{ml}$ methanol were vortexed for 30 minutes. The methanolic suspension was centrifuged, a small part of the supernatant diluted in assay buffer and directly analysed by EIA. Further on, we label the use of the diethyl ether extraction protocol with "Extraction", abbreviated "Extr". When no labellings are given we used the simplified method.

\section{Enzyme immunoassays (EIAs)}

All enzyme immunoassays used were already published, but not tested in horses. Details about the procedure and the cross-reactivities for the assays are published elsewhere (Palme and Möstl 1997; Möstl et al. 2002; Ganswindt et al. 2003). Interassay coefficients of variance for the assays in the horses were $7.8 \%$ and $20.9 \%(n=8)$ for the EIA measuring blood cortisol, and $13.3 \%$ and $15.7 \%(n=9)$ for the $3 \alpha, 11-$ oxo-A EIA measuring faecal GCMs. Values represent percentage variance for high and low quality controls. Details of the assays are shown in table 1. 
Table 1 Characteristics of the three EIAs in use for determining faecal glucocorticoid metabolites

\begin{tabular}{|c|c|c|c|}
\hline EIA & DOA EIA ${ }^{a}$ & $3 \alpha, 11-0 \times 0-A E^{\prime} A^{b}$ & $3 \alpha, 11 \beta$-dihydroxy-A EIAc \\
\hline $\begin{array}{l}\text { Antibody } \\
\text { against } \\
\text { (linked to } \\
\text { BSA) }\end{array}$ & $\begin{array}{l}\text { 11-oxoaetiocholanolone- } \\
\text { 3-HS }\end{array}$ & $\begin{array}{l}\text { 11-oxoaetiocholanolone- } \\
\text { 17-CMO }\end{array}$ & $\begin{array}{l}\text { 11ß-hydroxyaetiocholanolone-17- } \\
\text { CMO }\end{array}$ \\
\hline Label & $\begin{array}{c}\text { 11-oxoaetiocholanolone- } \\
\text { 3-glucorinide }\end{array}$ & $\begin{array}{l}\text { 11-oxoaetiocholanolone- } \\
\text { 17-CMOe }\end{array}$ & $\begin{array}{c}\text { 11ß-hydroxyaetiocholanolone-17- } \\
\mathrm{CMO}^{\mathrm{d}}\end{array}$ \\
\hline Standard & 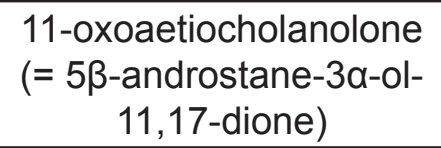 & 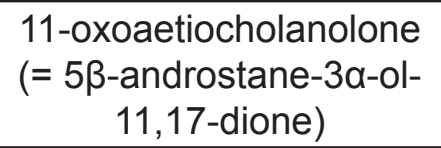 & 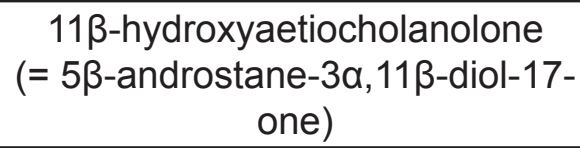 \\
\hline Specificity $^{f}$ & $11,17 \mathrm{DOA}^{\mathrm{g}}$ & $3 \alpha, 11-0 \times 0-\mathrm{CM}^{\mathrm{h}}$ & $3 \alpha, 11 \beta$-dihydroxy-CM ${ }^{i}$ \\
\hline
\end{tabular}

a First described by Palme and Möstl (1997)

${ }^{b}$ First described by Möstl et al. (2002)

c First described by Ganswindt et al. (2003)

d Coupled with N-biotinyl-1,8-diamino-3,6-dioxaoctane (DADOO-biotin)

e Coupled with biotinyl-3,6,9-trioxaundecanediamine (LC-biotin)

${ }^{f}$ Groups of metabolites measured

${ }^{g}$ CMs with 11,17-Dioxoandrostane configuration

${ }^{\mathrm{h}}$ CMs with $3 \alpha, 110 x 0$ configuration

' CMs with $3 \alpha, 11 \beta$-dihydroxy configuration

Concentration of immunoreactive metabolites after storage at room temperature

For stability analysis faecal samples were collected and from each sample one portion was frozen immediately whereas the other portions were frozen after storage for 1, 2, 4, 8 and 124 hours at room temperature. The content of GCMs was analysed using an EIA.

\section{High performance liquid chromatography (HPLC)}

The supernatant of methanolic suspension of faecal samples was separated by reversed phase high performance liquid chromatography (Novapac C18 column $0.39 \times 15 \mathrm{~cm}$, Fa. Waters, Milford, MA, USA). A linear methanol gradient from $50 \%$ to $75 \%$ in the first $40 \mathrm{~min}$ and thereafter $100 \%$ methanol up to 55 min were used. Flow rate was $1 \mathrm{ml} / \mathrm{min}$ and 3 fractions per minute were collected (95 fractions). Faecal samples of three animals were investigated. Elution positions of reference standards are shown in Fig. 4.

\section{Statistical analysis}

Data were analysed with the software package SPSS 15.0 for Windows. All statistical tests were two-tailed, and alpha was set at 0.05 . Spearman rank correlations were used for comparing the different extrac- tion methods and EIAs, as well as possible correlation between plasma cortisol and faecal GCMs. The baseline, highest and lowest values were tested for normality with Ks tests. As they do not significantly diverge from normality, we applied a paired t-test for their comparison.

\section{Results}

\section{Correlation between different assays}

The faecal samples of the ACTH stimulation test were analysed with three different EIAs (DOA EIA, 3a,11-oxo-A EIA and 3a,11ß-dihydroxy-A EIA), both using diethyl ether extraction or the supernatant of a methanolic suspension. When using methanolic supernatant, the values from DOA EIA significantly correlated with those of the $3 \alpha, 11-0 x 0-A$ EIA and the $3 \alpha, 11 \beta$-dihydroxy-A EIA (Spearman rank correlation; $n=43$; 3a,11-oxo-A EIA: $r=0.678, p<0.001 ; 3 \alpha, 11 \beta-$ dihydroxy-A EIA: $r=0.771, p<0.001)$. When using extraction, the values from DOA EIA significantly correlated with those of the $3 \alpha, 11 \beta$-dihydroxy-A EIA (Spearman rank correlation; $n=43 ; 3 \alpha, 11 \beta-$ dihydroxy-A EIA: $r=0.466, p=0.002$ ), but not with the results of the $3 \alpha, 11$-oxo-A EIA (Spearman rank correlation; $n=43$; 3a,11-oxo-A EIA: $r=0.109, p=$ 0.488). 


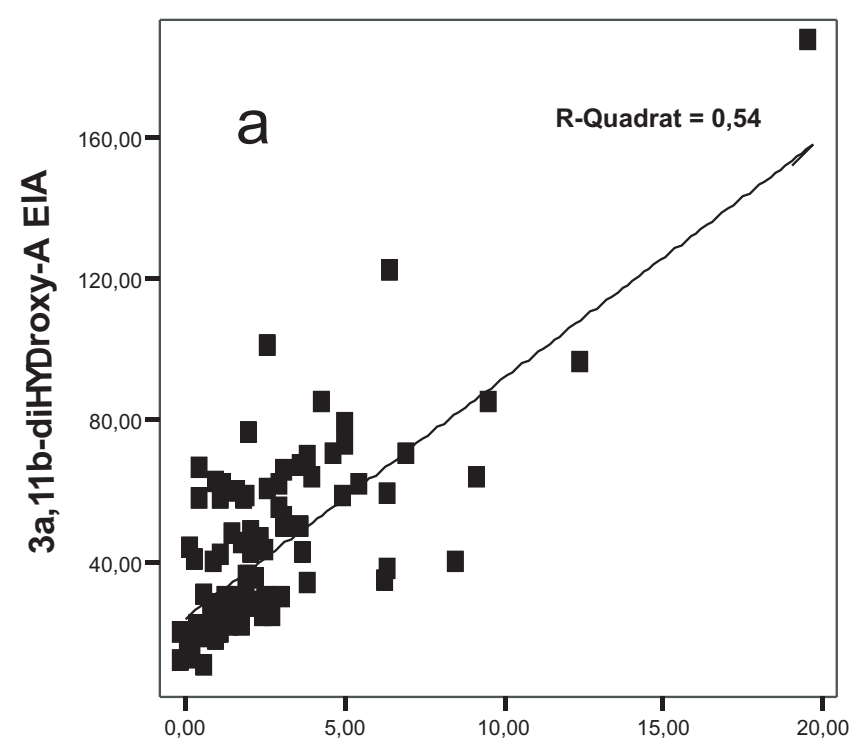

Fig. 1a-c Correlations between diethyl ether extractions of horse faeces analysed with DOA EIA on the $\mathrm{x}$-axis and supernatants of methanolic suspension of horse faeces analysed with different group-specific EIAs on the particular y-axis. (a) 3a,11ß-dihydroxy-A EIA, (b) DOA EIA and (c) $3 \alpha, 11-0 x 0-A$ EIA. Values represent concentrations of faecal GCMs in $\mathrm{ng} / \mathrm{g}$
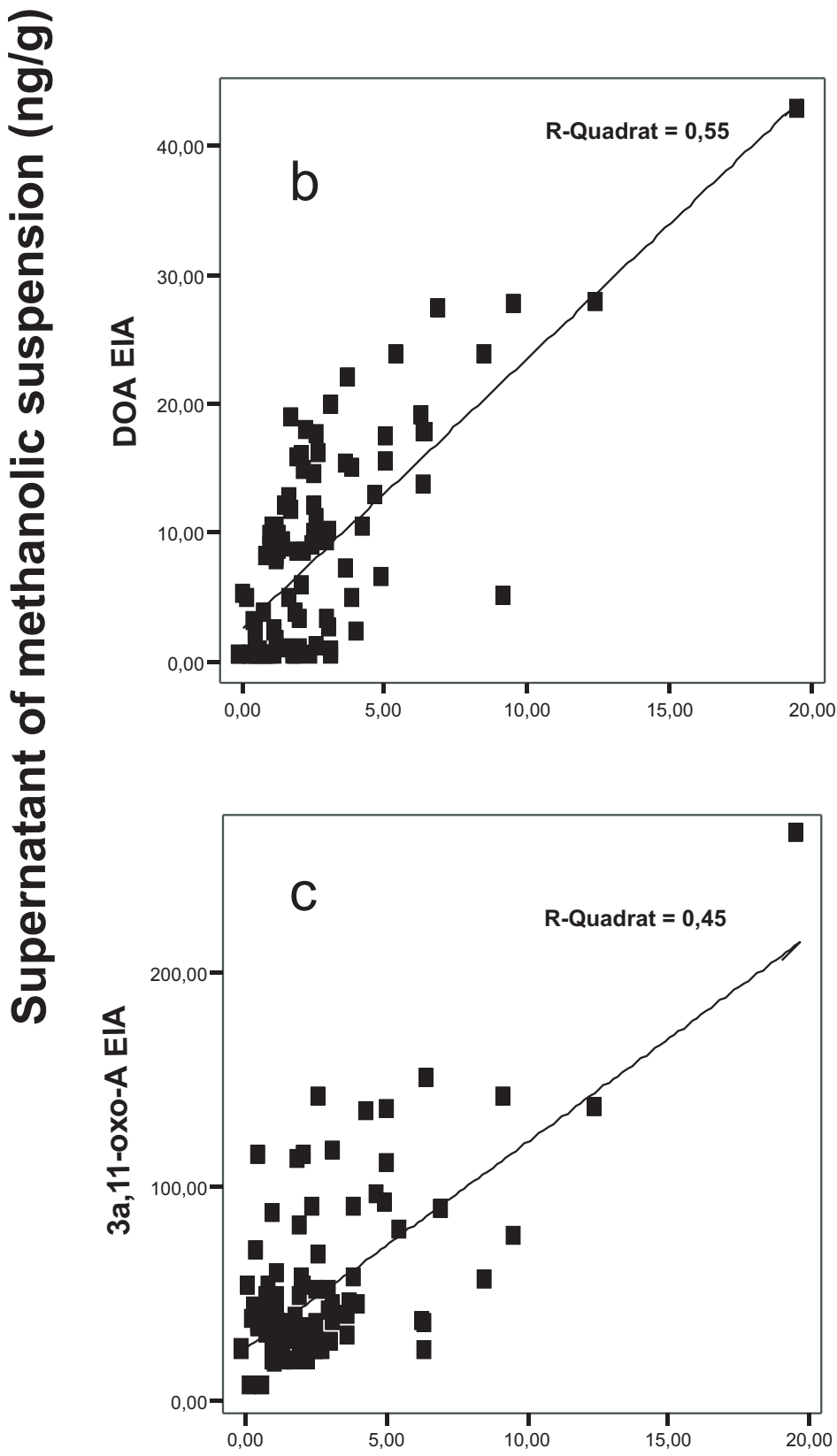

Diethyl ether extraction (DOA EIA, ng/g) 
As the data from different assays in general correlated within the same clean-up protocol, we compared data of the three different EIAs from samples treated with methanolic suspension to those from diethyl ether extraction. All assay values for the suspension method correlated highly significantly with values from DOA EIA when extracted with diethyl ether (Fig. 1). But $3 \alpha, 11 \beta$-dihydroxy-A EIA values and DOA EIA values showed a better correlation coefficient than 3a,11oxo-A EIA values to Extr-DOA EIA values (Spearman rank correlation; $n=88 ; 3 \alpha, 11 \beta$-dihydroxy-A EIA: $r$ $=0.640$; DOA EIA: $r=0.628$; 3a,11-oxo-A EIA: $r=$ 0.432; all $p<0.001$ ).

Correlation between mean plasma cortisol and mean faecal GCMs

For a better comparison between plasma cortisol and faecal GCMs we calculated the daily mean values for each parameter in the stimulation test. We compared the faecal GCM mean values from different assays

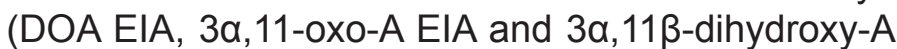
EIA) to plasma cortisol mean values. As horses excrete cortisol metabolites in faeces with a delay of about 24 hours (Palme et al. 1996; Möstl et al. 1999), we additionally shifted the correlation calculation between faecal cortisol metabolites and plasma cortisol for 1 and 2 days.

As expected, we could not find correlations between mean values of plasma cortisol to mean values of faecal metabolites for the same day. But for faeces collected one day later the correlation was highly significant for the analysis with $3 \alpha, 11-0 x 0-A$ EIA and ExtrDOA EIA, but slightly weaker for $3 \alpha, 11 \beta$-dihydroxy-A EIA. For the two day shift the correlations diminished again for all three assays. Details of the Spearman rank correlations (Spearman rank correlation coefficient, $\mathrm{p}$-values) are given in table 2.

Table 2 Correlation between mean plasma cortisol and mean faecal GCMs analysed with three different EIAs (Spearman rank correlations, $r=$ correlation coefficient, $p=$ significance value)

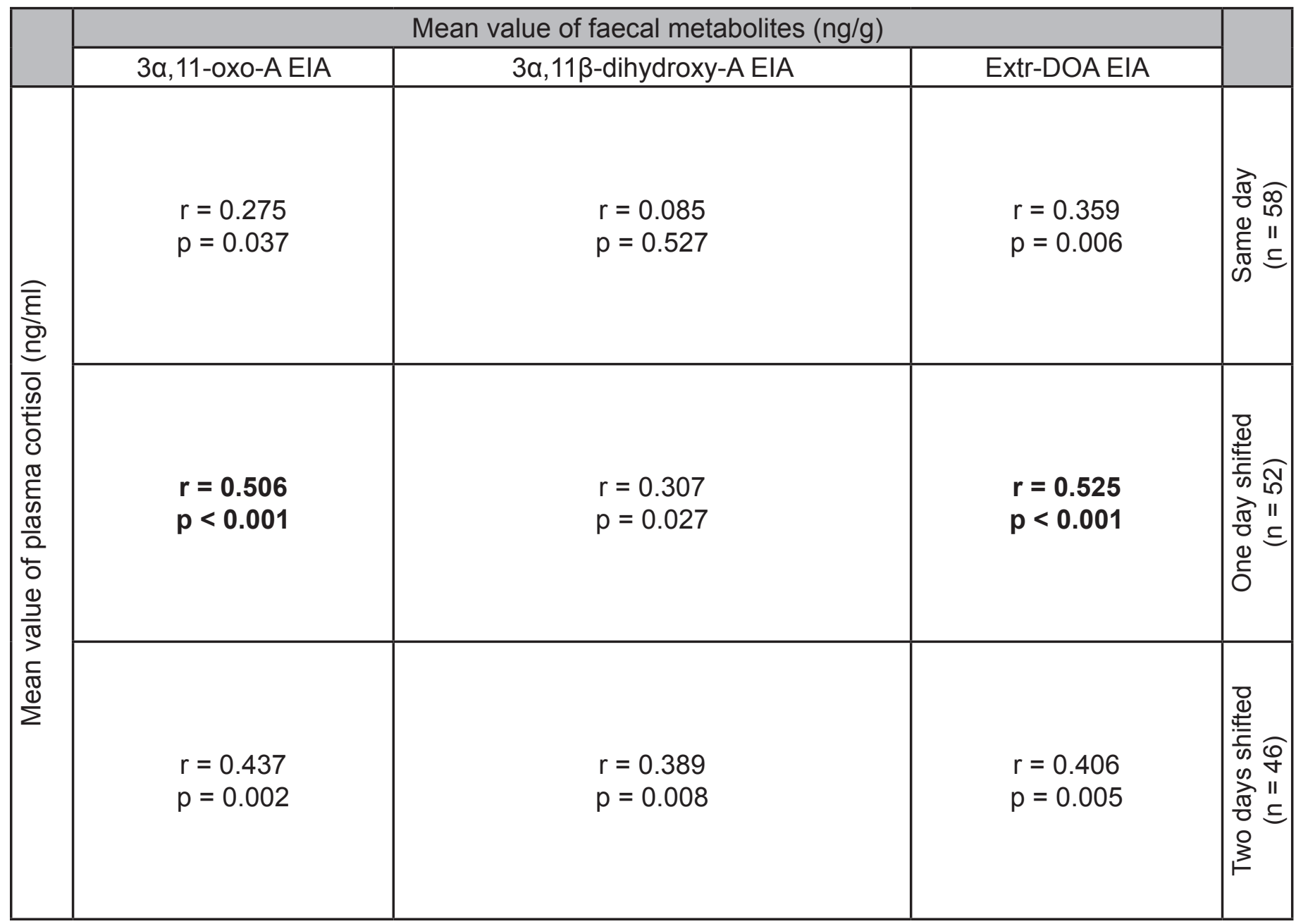


We calculated the means for the baseline, the highest and the lowest values during the procedure of ACTH stimulation and dexamethasone depression. For each horse the baseline values were averaged from the first three samples before stimulation, whereas the highest values were taken from the peak after ACTH stimulation and the lowest values from the depression after dexamethasone injection. In Fig. 2 faecal GCM concentrations of two horses during the ACTH Challenge Test, measured with 3a,11-oxo-A EIA and Extr-DOA EIA, are depicted.

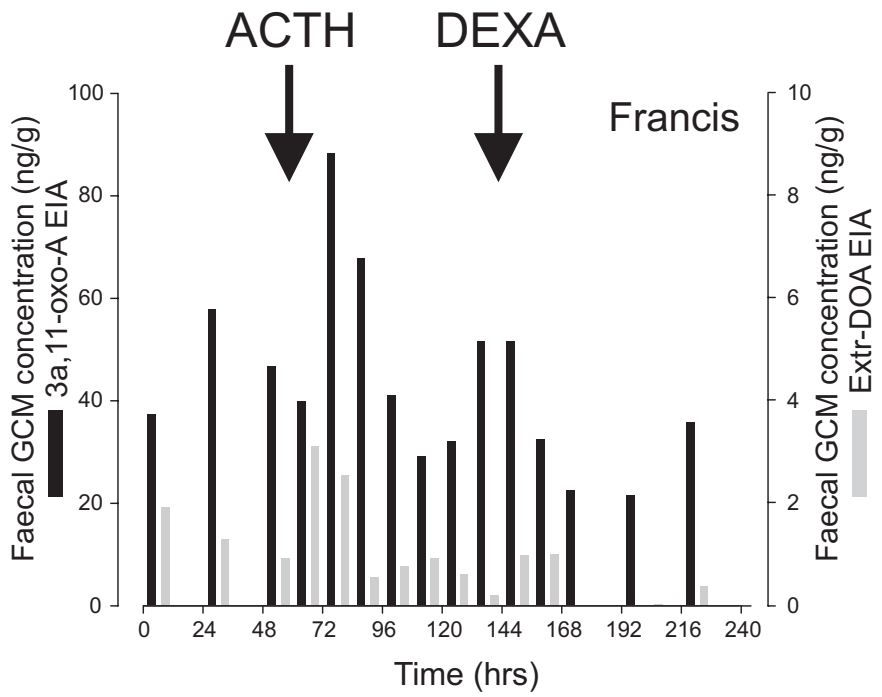

$34.07 \mathrm{ng} / \mathrm{g}(68 \%)$ for the assay $3 \alpha, 11$-oxo-A EIA values, which was highly significant (Paired t-test; see table 3), and for assay Extr-DOA EIA values the less significant mean decrease of $2.74 \mathrm{ng} / \mathrm{g}(78 \%)$.

\section{Stability analysis}

For stability analysis we compared the GCM content of immediately frozen faecal samples to those kept at room temperature for 1, 2, 4, 8 and 124 hours. All samples were analysed after methanolic suspension with either assay $3 \alpha, 11-0 x 0-A$ or assay DOA. The values from 3a,11-oxo-A EIA showed little variation

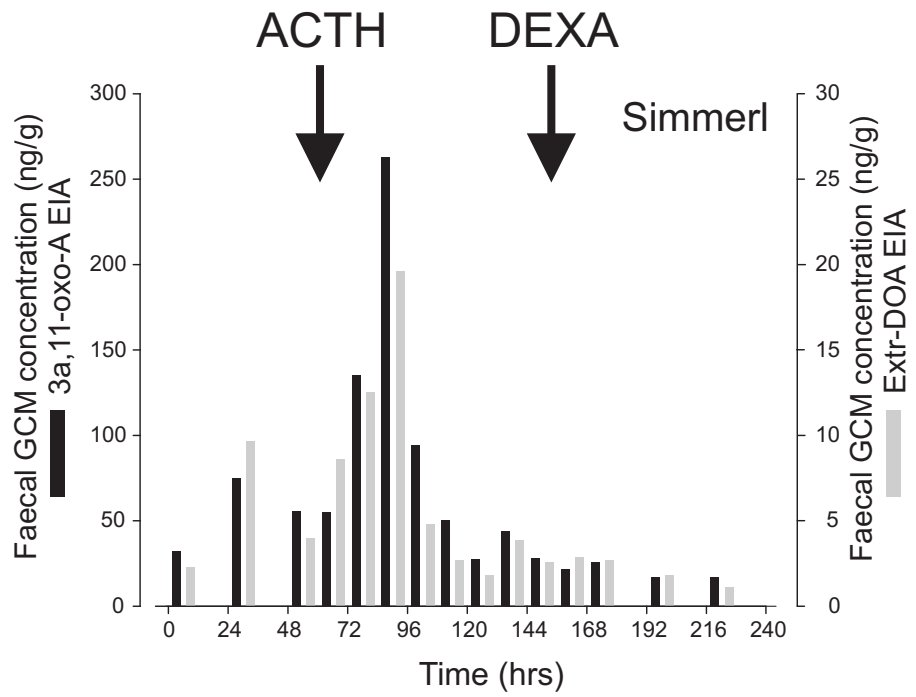

Fig. 2 Faecal GCM concentrations ( $\mathrm{ng} / \mathrm{g}$ ) in two horses during the ACTH stimulation and dexamethasone suppression test, analysed using 3a,11-oxo-A EIA and Extr-DOA EIA. Arrows indicate time point of ACTH/ dexamethasone application

We compared the assays for their amplitude range. Generally, higher quantities were measured using the 3a,11-oxo-A EIA than using the Extr-DOA EIA (mean baseline values: 3a,11-oxo-A EIA: $49.91 \pm$ $21.13 \mathrm{ng} / \mathrm{g}$; Extr-DOA EIA: $3.48 \pm 1.65 \mathrm{ng} / \mathrm{g}$, table $3)$. On average the difference between baseline and highest values increased by $55.18 \mathrm{ng} / \mathrm{g}(110 \%)$ for assay $3 \alpha, 11$-oxo-A EIA, which was significant (Paired t-test, see table 3). The mean increase of $4.76 \mathrm{ng} / \mathrm{g}$ $(140 \%)$ for the assay Extr-DOA EIA values was less significant. For the difference between baseline and lowest values we measured an average decrease of and a small standard deviation for up to 8 hours, but for those analysed with DOA EIA a strong variation could be seen ( $n=6$ for each time point, Fig. 3 ). In samples, which were stored for 124 hours at room temperature, the GCM concentration strongly declined for assay 3a,11-oxo-A, whereas for assay DOA the values showed a high variability (between $28 \%$ and $997 \%$ ) compared to the particular baseline values from the beginning of the test (Fig. 3, a single outlier at $997 \%$ is not depicted in the graph).

Table 3 Comparison of the amplitude range of the ACTH Challenge Test

\begin{tabular}{|c|c|c|c|c|c|c|c|c|c|}
\hline \multirow[t]{3}{*}{ assay } & \multirow[t]{3}{*}{$\begin{array}{c}\text { mean baseline values } \\
\pm \text { standard deviation } \\
\text { ng/g }\end{array}$} & \multirow[t]{3}{*}{$\begin{array}{c}\text { mean highest values } \\
\pm \text { standard deviation } \\
\text { ng/g }\end{array}$} & \multirow[t]{3}{*}{$\begin{array}{c}\text { mean lowest values } \\
\pm \text { standard deviation } \\
\mathrm{ng} / \mathrm{g}\end{array}$} & \multicolumn{3}{|c|}{$\begin{array}{c}\text { difference } \\
\text { baseline - high }\end{array}$} & \multicolumn{3}{|c|}{$\begin{array}{c}\text { difference } \\
\text { baseline - low }\end{array}$} \\
\hline & & & & \multirow[b]{2}{*}{$\mathrm{ng} / \mathrm{g}(\%)$} & \multicolumn{2}{|c|}{ t-test } & & \multicolumn{2}{|c|}{ t-test } \\
\hline & & & & & $\mathrm{n}$ & $p$ & $\mathrm{ng} / \mathrm{g}(\%)$ & $n$ & $p$ \\
\hline $\begin{array}{c}3 \alpha, 11-0 \times 0-A \\
\text { EIA }\end{array}$ & $49.91 \pm 21.13$ & $105.09 \pm 65.21$ & $15.84 \pm 9.28$ & $\begin{array}{c}55.18 \\
(110.56)\end{array}$ & 10 & 0.016 & $\begin{array}{c}34.07 \\
(68.26)\end{array}$ & 10 & $<0.001$ \\
\hline $\begin{array}{c}\text { Extr- } \\
\text { DOA EIA }\end{array}$ & $3.48 \pm 1.65$ & $8.24 \pm 6.03$ & $0.74 \pm 0.50$ & $\begin{array}{c}4.76 \\
(136.78)\end{array}$ & 6 & 0.062 & $\begin{array}{c}2.74 \\
(78.74)\end{array}$ & 6 & 0.005 \\
\hline
\end{tabular}



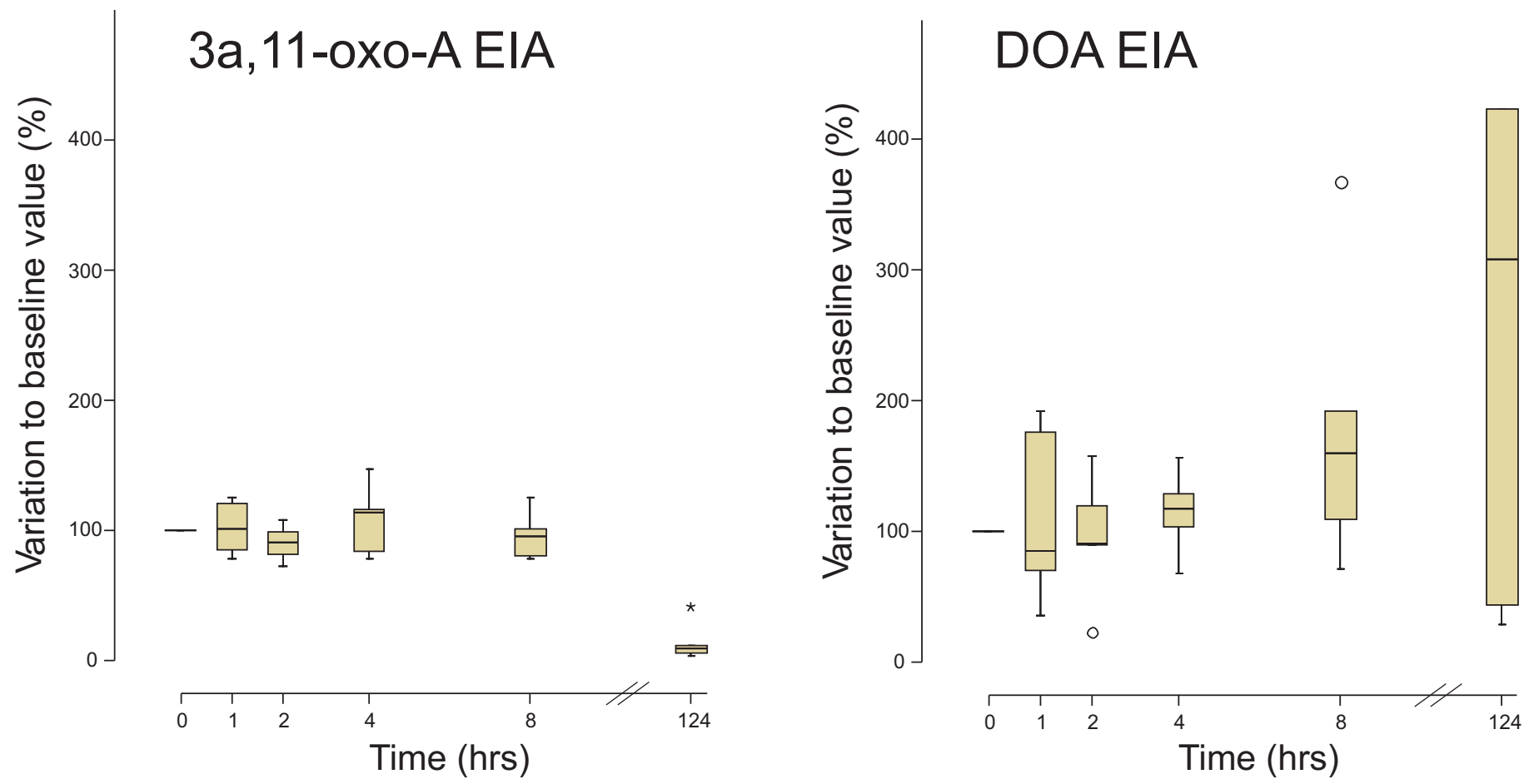

Fig. 3 Boxplot of the variation of GCMs (start time $=100 \%$ ) after different storage time at room temperature, analysed with $3 \alpha, 11-0 x 0-A$ EIA and DOA EIA, $n=6$ per time point. A single outlier (at $997 \%$ ) for the time point $124 \mathrm{hrs}$ analysed with DOA EIA is not depicted

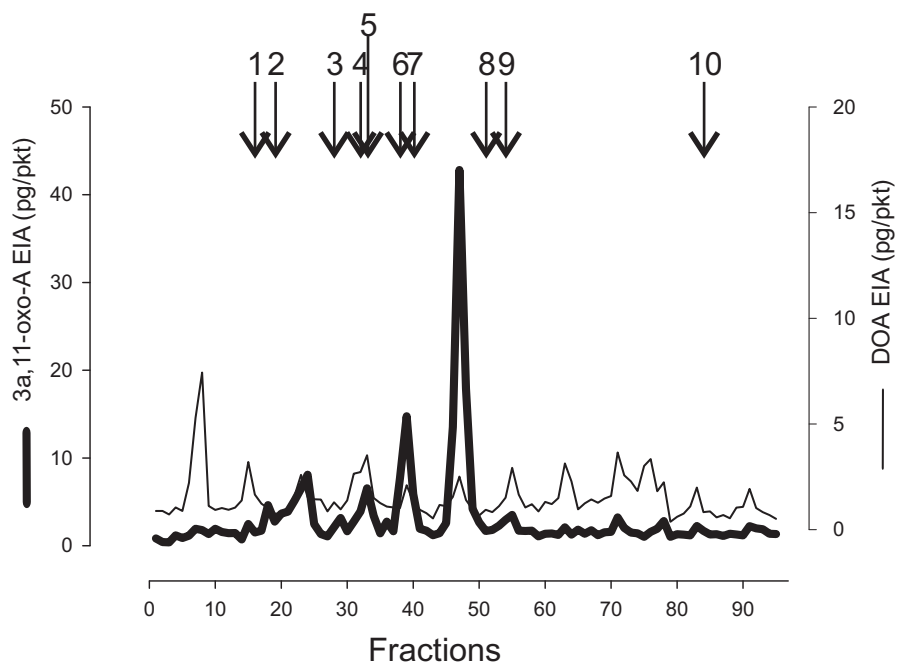

Fig. 4 High performance liquid chromatography (reversed phase) separations of immunoreactive faecal GCMs of a horse. The obtained fractions were analysed with DOA EIA and 3a,11-oxo-A EIA. Note the different scale of the y-axis. Elution positions of reference standards: 1) cortisone (fraction 16), 2) cortisol (19/20), 3) corticosterone (27-30), 4) 11ß-hydroxyaetiocholanolone $(32), 5)$ 11-oxoaetiocholanolone $(33), 6)$ tetrahydrocorticosterone (38), 7) androst-4-en-3,17dion (40), 8) testosterone (51), 9) 17a,20adihydroxyprogesterone $(54), 10)$ progesterone (84)

$\underline{\text { Immunoreactive metabolites }}$

The immunoreactive substances in the methanolic supernatant of faecal samples were separated by HPLC. The different fractions were analysed with DOA EIA and 3a,11-oxo-A EIA. The DOA EIA showed the dominating peak in fraction 8 , whereas the $3 \alpha, 11-0 x 0-A$ EIA showed a peak in fraction 39 and a second peak in fraction 47 (Fig. 4, one faecal sample is depicted as example). Both peaks were much higher than that measured with the DOA EIA. Also some minor peaks were present, amongst others one peak which coeluted like 11-oxoaetiocholanolone (fraction 33).

\section{Discussion}

The present study clearly proved that the $3 \alpha, 11$ oxo-A EIA using the simplified protocol of methanolic suspension correlates well with the established method for measuring GCMs in horse faeces, i.e. analysing the samples with DOA EIA after extraction (Merl et al. 2000). In addition, the 3a,11-oxo-A EIA has the advantage of providing a bigger difference between baseline values and peak values after ACTH stimulation, cross reacts with at least two other metabolites and in a higher extent than DOA EIA, which is shown by HPLC, and, the detected immunoreactive glucocorticoids are more stable at room temperature. The second EIA $(3 \alpha, 11 \beta$-dihydroxy-A) used, also gave good results after applying the methanolic suspension method. It correlates well with the esta- 
blished assay DOA EIA after extraction, but obtains inferior results than the $3 \alpha, 11$-oxo-A EIA concerning the correlation with mean plasma cortisol values.

Additionally, when compared to daily mean values of blood cortisol, daily mean values of faecal GCMs, after methanolic suspension, analysed with $3 \alpha, 11$ oxo-A EIA, produce correlation coefficients which are as good and significant as after extraction analysed with DOA EIA, both around $r=0.5$. The correlation is best when faecal samples are taken with one day delay. This is in agreement with Palme et al. (1996) and Möstl et al. (1999) findings of cortisol metabolites in horses being excreted via faeces about 24 hours delayed compared to plasma.

In general, higher values were found with the $3 \alpha, 11$ oxo-A EIA, which can be explained by having a closer look at the HPLC fractions measured with the DOA EIA and 3a,11-oxo-A EIA. Although both assays are designed to detect 11-oxoaetiocholanolone they differ in their linkage of the steroid for antibody production. 11-oxoaetiocholanolone is linked to BSA at position 3 in DOA EIA, and, in 3a,11-oxo-A EIA, to BSA at position 17. Some cross-reactions occur when the structure differences between cross-reacting molecules are located close to the linking position (Niswender and Midgley 1970; Kohen et al. 1975). As Möstl et al. (2002) already pointed out, the DOA EIA is group-specific concerning the oxo group in position 17 , whereas the $3 \alpha, 11-0 x 0-A$ EIA`s group specificity concerns the $\mathrm{OH}$-group in position 3 . With the $3 \alpha, 11$ oxo-A EIA two peaks were detected in the middle of the chromatogram, which were seen between the elution of tetrahydrocorticosterone and 17a,20adihydroxyprogesterone. 3a,11-oxo-A EIA obviously shows cross-reactions with $\mathrm{C} 21$ steroids, which are not detected with DOA EIA. Even though we did not conduct a radio metabolism study, it is likely that the detected metabolites are identical or closely related to the dominating GCMs present in horse faeces. These results highlight the importance of choosing an assay which is in good accordance with the metabolites excreted in a given species, and even between closely related species (Bosson et al. 2009).

The accuracy of faecal GCM measurements in general benefits from the cross-reaction characteristics of the 3a,11-oxo-A EIA, resulting in a higher baseline quantity as well as big amplitudes between baseline values and peak values after ACTH administration. The big amplitudes of 3a,11-oxo-A EIA strongly improve the practicability of GCM measurement in horses, because the higher the amplitudes the better small differences can be determined and the assay can be applied to different situations and to animals showing different levels of stress. It has to be mentioned that Extr-DOA values showed a higher incre- ase (in percentage) after ACTH administration than $3 \alpha, 11-0 x o-A$ values, but this method has not the overall benefit of high baseline quantities.

Another relevant aspect is the modification of GCMs after different storage time at room temperature. The time interval between defecation and freezing appears to be crucial in ruminants. Möstl et al. (1999) demonstrated a significant increase of $45 \%$ in horse faecal GCMs after 4 hours when measured with DOA EIA. We measured the stability of GCMs at room temperature with the assays $3 \alpha, 11-0 x 0-A$ and DOA. Obviously, the immunoreactive glucocorticoid values measured with 3a,11-oxo-A EIA are more stable compared to those measured with DOA EIA, showing less variation.

This method is superior to the assay used in horse faeces so far (application of DOA EIA after diethyl ether extraction) concerning the amplitude after ACTH induction and dexamethasone depression, as well as the stability of immunoreactive glucocorticoid metabolites. The simplified method poses big advantages, because it is faster and easier to apply, increases the accuracy and automatisation, but lowers the laboratory expenses (e.g. less consumption of solvent, feasible in simple equipped laboratories without pull-off devices) especially when adopted to huge quantities of faecal samples. The non-invasive sampling method, on the one hand, is particularly suitable when measuring stress in feral or wild horses for wildlife management, as well as conservation and behavioural biology. On the other hand, it is urgently needed for the growing sector of equestrian sports and the popular interest in the horse's welfare. With the simplified method higher amounts of samples can be analysed and the method has a better practicability which is necessary in determining the stress level of horses during sports events, e.g. after the transportation, during the contest itself or during the stay at the competition.

\section{Acknowledgments}

We thank Jürgen Heinze for helpful suggestions, Sandra Hake-Paulus, Petra Kölle, Anika Pachten, Bettina Wollanke (LMU Munich) and Hans-Peter Remler (LVFZ Haupt- und Landgestüt Schwaiganger Pferdehaltung) for assistance with conducting the experiment, Alexandra Kuchar and Andreas Trindl for technical assistance in the laboratory, and Henning Thies for language corrections. Horses have been provided by the LMU Munich and the LVFZ Hauptund Landgestüt Schwaiganger Pferdehaltung. The study was supported by an Excellence grant of the Universität Bayern e.V. and an HWP II grant of the University of Regensburg. 


\section{References}

Alexander S.L. and Irvine C.H.G. (1998) The effect of social stress on adrenal axis activity in horses: the importance of monitoring corticosteroid-binding globulin capacity. J Endocrinol 157:425-432.

Bosson C.O., Palme R., Boonstra R. (2009) Assessment of the stress response in columbian ground squirrels: laboratory and field validation of an enzyme immunoassay for fecal cortisol metabolites. Physiol Biochem Zool 82:291-301.

Cannon W.B. (1935) Stresses and strains of homeostasis. Am J Med Sci 189:1-14.

Ganswindt A., Palme R., Heistermann M., Borragan S., Hodges J.K. (2003) Non-invasive assessment of adrenocortical function in the male African elephant (Loxodonta Africana) and its relation to musth. Gen Comp Endocrinol 134:156-166.

Gorgasser I., Tichy A., Palme R. (2007) Faecal cortisol metabolites in Quarter Horses during initial training under field conditions. Vet Med Austria, Wien Tierärztl Mschr 94:226-230.

Heistermann M., Palme R., Ganswindt A. (2006) Comparison of different enzymeimmunoassays for the assessment of adrenocortical activity in primates based on fecal analysis. Am J Primatol 68:257-273.

Hopster H., van der Werf J.T.N., Erkens J.H.F., Blokhuis H.J. (1999) Effects of repeated jugular puncture on plasma cortisol concentration in loosehoused dairy cows. J Anim Sci 77:708-714.

Kohen F., Bauminger S., Lindner H.R. (1975) Preparation of antigenic steroid-protein conjugates. In: Cameron E.H.D., Hillier S.G., Griffiths K. (eds) Steroid Immunoassays. Omega Publishing, Cardiff, pp 11-32.

Matteri R.L., Carroll J.A., Dyer D.J. (2000) Neuroendocrine responses to stress. In: Moberg J.P. and Mench J.A. (eds) The Biology of Animal Stress. CABI Publishing, Oxford, pp 43-76.

Merl S., Scherzer S., Palme R., Möstl E. (2000) Pain causes increased concentrations of glucocorticoid metabolites in equine faeces. J Equine Vet Sci 20:586-590.
Moons C., Heleski C.R., Leece C.M., Zanella A.J. (2002) Conflicting results in the association between plasma and salivary cortisol levels in foals. Harvemeyer Foundation Workshop, Iceland.

Möstl E. and Palme R. (2002) Hormones as indicators of stress. Domest Anim Endocrinol 23:67-74.

Möstl E., Messmann S., Bagu E., Robia C., Palme R. (1999) Measurement of glucocorticoid metabolite concentration in faeces of domestic livestock. J Vet Med A 46:621-631.

Möstl E., Maggs J.L., Schrötter G., Besenfelder U., Palme R. (2002) Measurement of cortisol metabolites in faeces of ruminants. Vet Res Commun 26:127-139.

Niswender G.D. and Midgley A.R.Jr. (1970) In: Peron F.G. and Caldwell B.V. (eds) Immunologic Methods in Steroid Determination. Appleton-CenturyCrofts, New York, pp 149-173.

Palme R. and Möstl E. (1997) Measurement of cortisol metabolites in faeces of sheep as a parameter of cortisol concentration in blood. Int J Mamm Biol 62:192-197.

Palme R., Fischer P., Schildorfer H., Ismail M.N. (1996) Excretion of 14C-steroid hormones via faeces and urine in domestic livestock. Anim Reprod Sci 43:43-63.

Sapolsky R.M., Romero L.M., Munck A.U. (2000) How do glucocorticoids influence stress responses? Integrating permissive, suppressive, stimulatory and preparative actions. Endocr Rev 21:5589.

Selye H.A. (1936) A syndrome produced by diverse nocuous agents. Nature 138:32-33.

Touma C. and Palme R. (2005) Measuring fecal glucocorticoid metabolites in mammals and birds: the importance of validation. Ann N Y Acad Sci 1046:54-74.

Wingfield J.C. and Ramenofsky M. (1999) Hormones and the behavioural ecology of stress. In: Balm P.H.M. (ed) Stress Physiology in Animals. Sheffield Academic Press, pp 1-51. 